Rev. Saude pribl., S. Paulo

6: $431-3,1972$.

\title{
INFECÇÃO NATURAL DE FLEBOTOMINEOS EM FOCO ENZOÓTICO DE LEISHMANIOSE TEGUMENTAR NO ESTADO DE SÃO PAULO, BRASIL
}

\author{
Oswaldo Paulo FORATTINI ** \\ Dino B. G. PATTOLI ** \\ Emesto Xavier RABELLO ** \\ Octávio Alves FERREIRA ***
}

RSPSP-155

Forattini, O. P. et al. - Infecção natural de flebotomineos em foco enzootico de leishmaniose tegumentar no Estado de São Paulo, Brasil Rev. Saúde públ., S. Paulo, 6:431-3, 1972.

REsumo: Relata-se o encontro de infecçôes naturais de Pintomyia pessoai e Psychodopygus intermedius em foco enzoótico de leishmaniose tegumentar no Estado de São Paulo, Brasil. A natureza leishmaniótica desses encontros obteve confirmação através da inoculação experimental em hamsters.

UNITERMOS: Leishmaniose tegumentar enzootica *; Pintomyia pessoai *; Psychodopygus intermedius *; Infeccẫo nátural leishmaniotica de flebotomíneos.

No estado atual dos conhecimentos sobre a epidemiologia das leishmanioses tegumentares americanas, assume grande importância a pesquisa de infecçōes naturais de flebotomíneos por flagelados em forma de leptomonas (promastigotos). Vários relatos desses encontros limitaram-se à procura dos protozoários no tubo digestivo, associando-a a dados epidemiológicos locais. Todavia, a real natureza leishmaniótica dessas formas só poderá ser determinada através da execução de várias provas, principalmente, as tentativas de isolamento e de inoculaçōes experimentais.

Procuramos detectar a possivel presen- ça de infecção leishmaniótica nesses dípteros, procedendo a coletas de flebotomíneos no foco endêmico da Fazenda Jatai, Municipio de Luis Añtonio, Estado de São Paulo (Forattini et al.4, 1972). Para tanto, utilizamos dois métodos. O primeiro consistiu na formação de lotes, os quais, após prévia trituração em solução fisiológica, eram inoculados por via intradérmica, na região nasal de hamsters jovens. O segundo baseou-se na dissecção individual dos dípteros, segundo a técnica de Johnson et al. 5 (1963), seguida, em caso de positividade, de tentativa de isolamento em meio de NNN.

Os resultados obtidos até o momento, foram os seguintes:

Inoculaçōes - De maio de 1971 a fevereiro de 1972, foram coletados e inoculados em hamsters, 1452 flebotomíneos, distribuídos em oito lotes da seguinte maneira:

* Realizado com o auxílio parcial da FAPESP (Proc. C.+Médiças 70/788).

* Do Departamento de Epidemiologia da Faculdade de Saúde Pública da USP - Av. Dr. Arnaldo, 715 - São Paulo, SP, Brasil.

\#** Da Diretoria de Combate a Vetores da Superintendencia do Saneamento Ambiental (SUSAM) do Estado de Sð̃o Paulo - Rua Tamandaré, 649 - São Paulo, SP, Brasil. 
FORATTINI, O, P. et al. - Infeccão natural de flebotomíneos em foco enzótico de leishmaniose tegumentar no Estado de São Paulo, Brasil. Rev. Saúde públ., São Paulo, 6: 431-3, 1972.

\section{Espécie}

Psychodopygus intermedius

Pintomyia pessoai

Tolal

\begin{tabular}{rc} 
N. & Lotes \\
1273 & 7 \\
179 & 1 \\
\hline 1452 & 8
\end{tabular}

O conjunto constituído por espécimens de Pintomyia pessoai foi inoculado em 23/XI/1971. Um mês após, um dos animais apresentava pequeno nódulo local que, no entanto, revelou-se negativo ao exame direto por esfregaço. Essa lesão regrediu e, a 5/V/1972, notou-se a presença de pequena área de pelada com ligeira crosta. Nessa ocasião, o animal foi sacrificado e o exame histológico da região inoculada revelou a presença de formas em leishmania. O outro animal do lote continua em observação.

Em 22/II/1972, de um dos lotes de Psychodopygus intermedius, foram separados e inoculados 279 exemplares. Um dos animais sofreu o desenvolvimento de abscesso local que foi puncionado. $\mathrm{Em} 24 / \mathrm{IV} / 72$, esse hamster foi sacrificado revelando-se negativo ao exame histológico. O outro animal, em 14/VII/72, mostrou o desenvolvimento de lesão no local da inoculação, com o aspecto de pelada e crostas, sobre nódulo palpável (Figura). $O$ processo evoluiu e, a 8/IX/72, foi sacrificado. O exame histologico revelou a presença de leishmanias e do material retirado foi feita nova inoculação direta, em outro lote, que está em observação.

Quanto às demais inoculações, se bem que em vários hamsters tenhă sido observada a formação de nódulos, não foi possível, até o momento, detectar a presença da infecção. Os animais inoculados continuam em observação.

Dissecções - De janeiro a março de 1972, foram dissecados 160 exemplares de Psychodopygus intermedius. Observou-se um resultado positivo em 22/II, com formas em leptomonas (promastigotos) no tubo digestivo, e correspon-

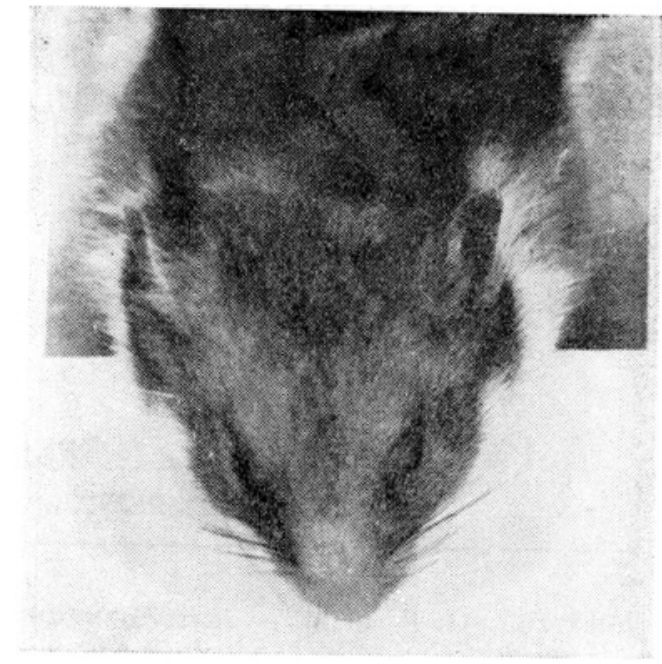

Figura: Lesão nodular crostosa, positiva para formas em leishmania (amastıgotos), provocada. em hamster pela inoculaçăo de Psychodopygus intermedius naturalmente infectado. Aspecto após cerca de 5 meses de evolução.

dendo a conjunto de exemplares retirados do mesmo lote supracitado e que foi inoculado em hamsters na mesma data. O material foi imediatamente suspenso em solução fisiológica, contendo 500 unidades de penicilina $G$ potássica cristalina e $1 \mathrm{mg}$ de sulfato de estreptomcina por ml. A suspensão foi semeada em meio de NNN, tendo-se obtido o isolamento da amostra.

Em seguida, procedeu-se à inoculação experimental, tendo-se utilizado seis 1otes de hamsters, da seguinte maneira:

$\begin{array}{cc}\text { Repique } & \text { Lotes } \\ 1^{\circ} & 2 \\ 2^{\circ} & 2 \\ 4^{\circ} & 1 \\ 5^{\circ} & 1\end{array}$

Em várias oportunidades, verificou-se - desenovlvimento de nódulos e peladas na regiāo inoculada.

Em 20/X/72, um dos animais inoculado com o $5 .^{\circ}$ repique apresentou nódulo evi- 
FORATTINI, O. $P$. et al. - Infecção natural de flebotomíneos em foco enzoótico de leishmaniose tegumentar no Estado de São Paulo, Brasil. Rev. Saúde públ., São Paulo, 6: 431-3, 1972.

dente no ponto de inoculação. Em $17 / \mathrm{XI} / 72$, essa formação havia evoluído de maneira evidente, quando foi retirado material por meio de punção. $O$ exame dos esfregaços, corados pelo método de Giemsa, revelou a presença de raras formas em leishmania (amastigotos). Os outros animais desse lote desenvolveram também lesões nodulares. Todavia, até o momento, não foi possivel evidenciar a natureza leishmaniótica dessas lesões, continuando em observação.

Em épocas passadas, no Estado de São Paulo, tanto Pintomyia pessoai como Psychodopygus intermedius foram assinalados com infecção natural por leptomonas. Contudo, o seu carater leishmaniotico foi apenas suspeitado, face aos elementos epidemiológicos locais (Aragĩo 1, 1922; Pessôa e Coutinho 6, 7, 1940, 1941; Coutinho 2,1940 \& Foratrini e SANTOS $^{3}$, 1952). Com os achados relatados nesta nota, confirma-se o possível papel vetor desses flebotomíneos.

RSPSP-155

Forattins, O. P. et al. [Natural infections of Phlebotominae sandflies in $a$ enzootic focus of cutaneous leishmaniasis in S. Paulo State, Brazill. Rev. Saúde públ., S. Paulo, 6:431-3, 1972.

Summary: Natural infections of Phlebotominae sandflies Pintomyia pessoai and Psychocopygus intermedius in $a$ sylvatic enzootic focus of cutaneous leishmaniasis in S. Paulo State, Brazil, are reported. For both species confirmation was obtained by experimental inoculation in hamsters.

UNITERMS: Enzootic cutaneous leishmaniasis *; Pintomyia pessoai *; Psychodopygus intermedius *; Natural leishmanial infections of Phlebotominae.

\section{REFERENCIAS BIBLIOGRAFICAS}

1. ARAGäO, H. de B. - Transmissão da leishmaniose no Brasil pelo Phlebotomus in. termedius. Bras. med., 36: 129.30, 1922.

2. COUTINHO, J. O. - Localização de formas em leptomonas, possivelmente de Leishmania brasiliensis, na faringe de Phlebotomus pessoai naturalmente infectado An. Fac. Med. S. Paulo, 16: 163-71, 1940.

3. FAROTTNI, O. P. \& SANTOS, M. R. dos - Nota sôbre infecção natural de Phle. botomus intermedius Lutz e Neiva, 1912, por formas em leptomas, em um foco de leishmaniose tegumentar americana. Arq. Hig., S. Paulo, 17: 171-4, 1952.

4. Forattini, O. P. et al. - Nota sobre um foco de leishmaniose tegumentar na região nordeste do Estado de São Paulo, Brasil. Rev. Saúde púb., S. Paulo, 6: 103.5, 1972 .

5. JOHNSON, P. T. et al. - Natural infections of leptomonad flagellates in Panamanian Phlebotomus sandflies. Exper. Parasit., 14: 107-22, 1963.

6. PESSOA, S. B. \& COUTINHO, J. O. Infeção natural de Phlebotomus pessoai por formas em leptomonas, provavelmente em Leishmania brasiliensis. Rev. Biol., Hig., 10: 139-42, 1940.

7. PESSóA, S. B. \& COUTINHo, J. O. Infecção natural e experimental dos flebótomos por Leishmania brasiliensis, no Estado de São Paulo. Hospital, Rio de Janeiro, 20: 25-35, 1941.

Recebido para publicação em 22.11-1972

Aprovado para publicąão em 26.11-1972 